\title{
Ajuste e avaliação dos modelos agregados de estimativas de custo de PCHs no Brasil
}

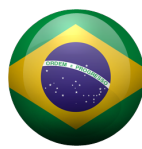 \\ Ivan Felipe Silva dos Santos \\ Engenheiro Hídrico graduado pela UNIFEI. Grupo de Energia Renováveis (GEER). Itajubá [Minas Gerais] \\ Brasil. <ivanfelipedeice@hotmail.com>.

\section{Geraldo Lucio Tiago Filho} \\ Eng. Mecânico, Doutor (USP) e Mestre (UNIFEI). Prof. Dr. (IRN / UNIFEI). Grupo de Energia Renováveis \\ (GEER). Itajubá [Minas Gerais] Brasil. <tiagounifei@hotmail.com>.

\section{Regina Mambeli Barros}

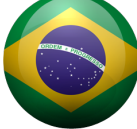 \\ Eng. Civil, Doutora e Mestre (PPG-SHS/EESC/USP). Profa. Dra. (IRN / UNIFEI). Grupo de Energia \\ Renováveis (GEER). Itajubá [Minas Gerais] Brasil. <remanbeli@hotmail.com>.

\section{Helmo Lemos}

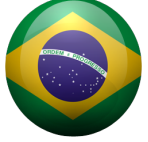 \\ ngenheiro Civil e Economista. Pesquisador (CERPCH / UNIFEI). Grupo de Energia Renováveis (GEER) \\ Itajubá [Minas Gerais] Brasil. <helmo_CERPCH@hotmail.com>.
}

\section{Resumo}

A viabilidade de empreendimentos de geração hidrelétrica deve ser testada antes da construção do mesmo. Para isto são necessárias estimativas de custo, que normalmente são construídas a partir de dados históricos. Contudo a construção destes modelos, com precisão satisfatória, pode se tornar difícil e até mesmo impossível, devido às particularidades técnicas, ambientais e sociais de cada projeto. Neste contexto diversos autores têm desenvolvido equações para determinação de custos de Pequenas Centrais Hidrelétricas em diversas regiões do mundo. 0 presente artigo analisa e compara, por meio de gráficos e parâmetros estatísticos, três metodologias de estimativas de custo aplicadas ao cenário Brasileiro com os dados locais de custos de empreendimentos reais. $\mathrm{O}$ impacto das diferentes abordagens sobre os benefícios e o ponto ótimo de um projeto de implantação de uma PCH também foi verificado. Os resultados obtidos foram analisados e discutidos.

\section{Palavras-chave}

Pequenas Centrais Hidrelétricas (PCH); estimativa de custos; viabilidade econômica; comparação.

\section{Setting and assessment of aggregate models for SHP's cost estimates in Brazil}

\begin{abstract}
The feasibility of hydroelectric generation projects should be tested before the construction of the same. For this are necessary cost estimates, which are usually constructed from historical data. However the construction of these models, with satisfactory precision, can become difficult and even impossible, due to the technical, environmental and social particularities of each Project. In this context several authors have developed equations for determining costs of Small Hydropower in various regions of the world. This article analyzes and compares, using graphical and statistical parameters, three different methodologies for estimating costs applied to the Brazilian scenario with local cost data from actual enterprises. The impact of different approaches on the benefits and on the optimal point of a project to establish a PCH was also checked. The results were analyzed and discussed.
\end{abstract}

\section{Keywords}

Small Hydro Power (SHP); cost estimating; economic feasibility; comparison. 


\section{Introdução}

Mason et al. (2010) afirma que o aumento da geração de energia elétrica por meio de fontes renováveis é a chave para a urgente e necessária diminuição de emissões de gases de efeito estufa na atmosfera. Neste contexto, a energia hidráulica, fonte de energia renovável e limpa, se torna uma opção bastante importante.

As divisões entre as classes de centrais hidrelétricas e o critério para classificação de $\mathrm{PCH}(\mathrm{s})$ (Pequenas Centrais Hidrelétricas) não é uniforme entre os países, podendo, segundo Zhang et al. (2012), variar de $\mathrm{P}<1$ [MW] na Alemanha a P < 50 [MW] no Canada. De acordo com ANEEL (2003), no Brasil as PCHs são aquelas cuja potência de aproveitamento está limitada entre: 1 [MW] e 30 [MW]. Nestas centrais, o nível da água a jusante da casa de máquinas deve ser determinado sem considerar a vazão vertida e a área do reservatório das $\mathrm{PCH}(\mathrm{s})$ deve ser inferior a $13\left[\mathrm{~km}^{2}\right]$ obedecendo a inequação:

$$
A \leq \frac{14,3 P}{H b}
$$

Onde: $A=$ área do reservatório $\left[\mathrm{km}^{2}\right], P=$ Potência a ser instalada $[M W], e$ $\mathrm{Hb}=$ Queda bruta $[\mathrm{m}]$.

Como o custo de uma PCH é um fator limitante a sua implantação, a estimativa deste é importante por permitir uma análise prévia do tempo de retorno do investimento e da viabilidade do empreendimento. Neste contexto, vários autores têm estudado estimativas de custo de PCHs para várias regiões do mundo, tais como: Kaldellis et al. (2005), Singal e Saini (2007), Souza et al. (2009), Santos et al. (2010), Aggidis et al. (2010), Tiago filho et al. (2013), etc. Contudo estas estimativas podem ser de difícil construção, e até mesmo impossível, devido as particularidades técnicas, ambientais e sociais de cada projeto, que podem elevar o custo deste e fazer com que se afaste da sua tendência regional.

De acordo com Souza et al. (2009), a estimativa dos custos de centrais hidrelétricas podem ser realizadas utilizando-se modelos agregados ou desagregados. 0 primeiro, parte do pressuposto que o custo total do projeto é função da potência e da queda bruta da central. Já o segundo, estima o custo total por meio da soma dos custos dos componentes individuais, obtidos a partir de suas características próprias principais.

O principal objetivo do presente trabalho é comparar custos de projetos reais aos custos calculados por meio de três modelos agregados de estimativas do cenário Brasileiro. Os benefícios econômicos de uma PCH também serão determinados por meio destes três modelos a fim de que se possam analisar quais os impactos destes em um projeto real.

\section{Modelos de estimativas de custo analisadas}

\subsection{Modelo apresentado por Tiago Filho et al. (2011)}

0 modelo apresentado por Tiago filho et al. (2011) consiste na correlação dos custos unitários (Cun) com o fator de aspecto FA da PCH. Fator este que decorre da equação utilizada para determinação da rotação especifica da turbina selecionada para determinada central hidrelétrica (Equação 2):

$$
n_{q a}=1000 \cdot n \cdot \frac{Q^{0,5}}{(g \cdot H)^{0,75}}
$$

Onde: $n=$ rotação da turbina hidráulica [rpm], $Q=$ vazão turbinada $\left[\mathrm{m}^{3} / \mathrm{s}\right]$, $g=$ aceleração da gravidade $=9,81\left[\mathrm{~m}^{2} / \mathrm{s}\right]$ e $H=$ queda bruta na qual a turbina está submetida $[\mathrm{m}]$ e

$n_{q a}=$ rotação especifica definida como a rotação que a maquina possuiria se operasse a um metro de queda com $1\left[\mathrm{~m}^{3} / \mathrm{s}\right]$ de vazão. 
Dividindo-se nqa por $\mathrm{n}$ na equação 1, e substituindo a relação entre potência e vazão (Equação 3), obtemos uma função da potência e da queda que nomeamos fator de aspecto FA (Equação 4):

$$
\begin{aligned}
& Q=\left(\frac{P \cdot 1000}{g \cdot H}\right) \\
& \frac{n_{q a}}{n}=1821,43 \cdot \frac{p^{0,5}}{H^{1,25}}=F A
\end{aligned}
$$

Onde: $P=$ Potência $[M W], \gamma=$ Peso especifico da água $=9800\left[\mathrm{~N} / \mathrm{m}^{3}\right]$ e $F A=$ fator de aspecto [rpm-1].

Sendo:

$$
\operatorname{Cun}\left[\frac{\$}{k W}\right]=k \cdot(F A)
$$

Onde: $K$ e $n$ são constantes que variam de acordo com as características do mercado da região de estudo.

Observa-se, pela Equação 3, que além de o fator de aspecto (FA) se relacionar com a rotação e rotação especifica das maquinas, que são escolhidas em função do aspecto físico da central, ele também pode ser escrito como uma função que varia com os valores de $\mathrm{H}$ - P escolhidos para implantação da central hidrelétrica em um local potencial, fatores estes que também estão estritamente relacionados ao formato físico da central, e são preponderantes na determinação dos custos das obras civis e das turbinas a serem escolhidas. Desta forma conclui-se que o fator FA pode ser relacionado com os custos totais de projetos de hidrelétricas. Tiago Filho et al. (2011) utilizou ainda dados de projetos Brasileiros a fim de determinar a relação do FA com os custos de PCHs nesta região, apresentada na Equação 6.

$$
\operatorname{Cun}\left[\frac{U S \$}{k W}\right]=1654 .(F A)^{0,085}
$$

\subsection{Modelo apresentado por Souza et al. (2009)}

O modelo apresentado por Souza et al. (2009) parte do principio que o custo de uma central hidrelétrica é proporcional a sua potência e inversamente proporcional a sua queda bruta, sendo escrito em função de uma constante k estimada em função de projetos anteriores na região de interesse, conforme demonstra a Equação 7.

$$
C_{t o t}\left[10^{6} \text { US } \$=\frac{k \cdot P[M W]^{0,82}}{H^{0,246}}\right.
$$

Utilizou-se para determinação da constante k no cenário Brasileiro os valores de custos reais de projetos de PCHs Brasileiras, obtidos junto a um escritório de engenharia e apresentados na Tabela 1. Igualou-se o Ctot da Equação 6, aos valores observados em projetos da Tabela 1 e calculou-se então a série de valores de $\mathrm{k}$ que mais aproximavam a estimativa em questão dos valores reais. Estes valores de $\mathrm{k}$ foram relacionados com a potência das centrais, o que resultou em um coeficiente de correlação satisfatório $\left(R^{2}=0,851\right)$ conforme apresenta a Figura 1 . 
Tabela 1. Dados de projetos do cenário Brasileiro.

\begin{tabular}{|c|c|c|c|c|c|}
\hline PCH & P [MW] & $\mathbf{Q}\left[\mathrm{m}^{3} / \mathrm{s}\right]$ & $\mathbf{H}[\mathbf{m}]$ & $\begin{array}{c}\text { Investimento Total } \\
\text { [10 } 0^{6} \text { US } \$\end{array}$ & [US\$/kW] \\
\hline PCH 1 & 21,30 & 245,40 & 10,16 & 54,5 & 2560,10 \\
\hline PCH 2 & 16,00 & 253,00 & 7,48 & 36,3 & 2267,14 \\
\hline PCH 3 & 17,70 & 172,95 & 12,23 & 72,6 & 4104,41 \\
\hline PCH 4 & 24,50 & 245,40 & 11,90 & 95,4 & 3893,83 \\
\hline PCH 5 & 1,00 & 0,75 & 162,90 & 1,9 & 1857,92 \\
\hline PCH 6 & 1,00 & 6,00 & 20,37 & 1,1 & 1143,35 \\
\hline PCH 7 & 5,90 & 11,35 & 60,14 & 9,2 & 1554,44 \\
\hline РCH 8 & 2,90 & 3,11 & 107,70 & 9,0 & 3095,18 \\
\hline PCH 9 & 6,30 & 4,85 & 153,26 & 9,9 & 1574,59 \\
\hline PCH 10 & 4,00 & 10,25 & 46,56 & 10,9 & 2728,53 \\
\hline PCH 11 & 3,40 & 10,50 & 38,12 & 7,2 & 2106,12 \\
\hline PCH 12 & 3,60 & 9,50 & 44,11 & 7,5 & 2094,54 \\
\hline PCH 13 & 1,00 & 11,25 & 10,58 & 3,6 & 3644,84 \\
\hline PCH 14 & 8,00 & 43,00 & 21,60 & 18,5 & 2311,33 \\
\hline PCH 15 & 7,00 & 51,83 & 15,00 & 14,8 & 2118,22 \\
\hline PCH 16 & 4,60 & 12,45 & 39,00 & 9,7 & 2107,39 \\
\hline PCH 17 & 6,20 & 9,50 & 70,50 & 15,4 & 2491,13 \\
\hline PCH 18 & 7,00 & 28,00 & 30,00 & 13,3 & 1906,90 \\
\hline PCH 19 & 4,50 & 60,25 & 9,00 & 10,8 & 2389,16 \\
\hline PCH 20 & 5,00 & 26,25 & 22,00 & 12,2 & 2438,42 \\
\hline PCH 21 & 5,00 & 24,30 & 23,80 & 12,2 & 2438,42 \\
\hline
\end{tabular}

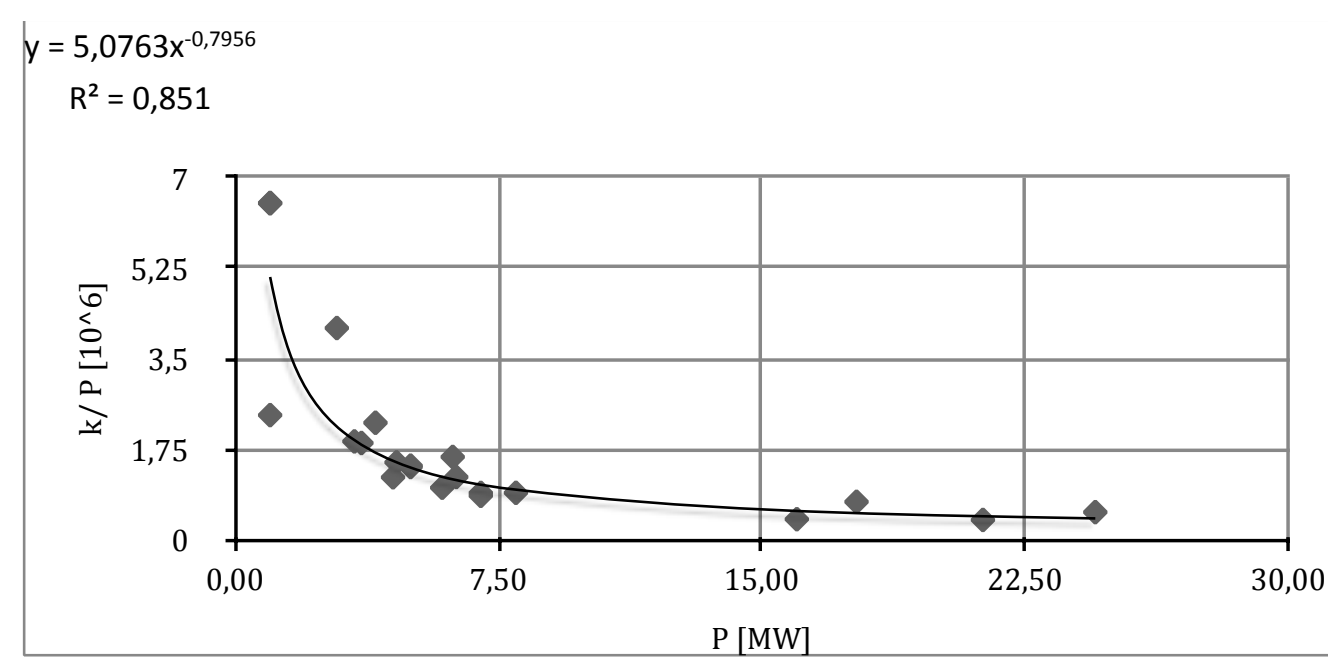

Figura 1. Determinação da constante k para estimativa de custos no Brasil. 
Desta forma a estimativa de Souza et al. pode ser aplicada ao mercado Brasileiro por meio da Equação 8:

$$
C_{\text {tot }}\left[10^{6} U S \$\right]=\frac{5,076 . P[M W]^{1,03}}{H^{0,246}}
$$

O método desenvolvido por Kaldellis et al. (2005) foi originalmente desenvolvido para usinas construídas na Grécia, conforme apresenta a Equação 9.

$$
\operatorname{Cun}\left[\frac{U S \$}{k W}\right]=(1+\xi) \cdot 4484,7 \cdot P[k W]^{-0,122} \cdot H^{-0,107}
$$

Onde $\xi$ é uma constante, que varia de acordo com o mercado local, assumindo valores entre 0,3 e 1. Os dados históricos de custos unitários de PCHs Brasileiras (apresentados na tabela 1) foram então mais uma vez utilizados a fim de que se ajustasse uma constante $\xi$ representativa do cenário Brasileiro.

0 ajuste foi realizado por meio do método dos mínimos quadrados. Este consiste na minimização entre o desvio entre a grandeza observada e calculada conforme apresenta a Equação 9. 0 método foi aplicado por meio do aplicativo Solver do software Microsoft Excel@, que permitiu a obtenção do $\xi$ que melhor ajustava a estimativa de Kaldellis et al. (2005) ao cenário Brasileiro, a saber, $\xi=1$.

$$
\min D, \quad D=\left(C u n_{\text {obs }}-C u n_{\text {calc }}\right)^{2}
$$

\section{Resultados}

Utilizando-se os dados da Tabela 1 pode-se aplicar e comparar os custos reais de projetos de PCHs no Brasil com os custos estimados pelos modelos supracitados. Esta comparação está apresentada na Tabela 2, onde D1, D2 e D3 os módulos dos desvios relativos percentuais entre cada uma das estimativas (Conforme a ordem adotada no tópico 2) e os valores observados e em gráfico, nas Figuras 2 e 3.

Tabela 2. Custos estimados e desvios relativos.

\begin{tabular}{|c|c|c|c|c|c|c|}
\hline $\begin{array}{c}\text { Ctot Observado } \\
(\mathbf{1 0} \text { ( US\$) }\end{array}$ & $\begin{array}{c}\text { Ctot - } \\
\text { Tiago Filho } \\
\text { et al. (2011) }\end{array}$ & D1 [\%] & $\begin{array}{c}\text { Ctot - } \\
\text { Souza } \text { et al. } \\
\mathbf{( 2 0 1 1 )}\end{array}$ & D2 [\%] & $\begin{array}{c}\text { Ctot - } \\
\text { Kaldellis } \text { et al. } \\
\text { (2005) }\end{array}$ & D3 [\%] \\
\hline 54,53 & 59,36 & 8,86 & 67,00 & 22,86 & 44,19 & 18,96 \\
\hline 36,27 & 45,51 & 25,46 & 53,80 & 48,32 & 35,52 & 2,08 \\
\hline 72,65 & 47,99 & 33,94 & 52,90 & 27,19 & 36,82 & 49,31 \\
\hline 95,40 & 67,55 & 29,20 & 74,44 & 21,97 & 49,13 & 48,50 \\
\hline 1,86 & 1,82 & 1,91 & 1,45 & 21,95 & 2,24 & 20,52 \\
\hline 1,14 & 2,27 & 98,80 & 2,42 & 111,51 & 2,80 & 144,64 \\
\hline 9,17 & 12,89 & 40,55 & 11,53 & 25,72 & 11,84 & 29,05 \\
\hline 8,98 & 5,78 & 35,63 & 4,81 & 46,45 & 5,96 & 33,59 \\
\hline 9,92 & 12,50 & 25,97 & 9,80 & 1,21 & 11,34 & 14,35 \\
\hline
\end{tabular}

SANTOS, I.F.S.; TIAGO FILHO, G.L.; BARROS, R.M.; LEMOS, H.. Ajuste e avaliação dos modelos agregados de estimativas de custo de PCHs no Brasil. Labor \& Engenho, Campinas [SP] Brasil, v.9, n.3, p.13-22, jul./set. 2015. 


\begin{tabular}{|c|c|c|c|c|c|c|}
\hline 10,91 & 8,83 & 19,07 & 8,23 & 24,61 & 8,65 & 20,77 \\
\hline 7,16 & 7,62 & 6,36 & 7,31 & 2,10 & 7,66 & 6,97 \\
\hline 7,54 & 7,96 & 5,56 & 7,48 & 0,79 & 7,93 & 5,16 \\
\hline 3,64 & 2,44 & 33,14 & 2,84 & 22,05 & 3,00 & 17,69 \\
\hline 18,49 & 19,74 & 6,76 & 20,30 & 9,77 & 17,25 & 6,69 \\
\hline 14,83 & 17,85 & 20,41 & 19,35 & 30,49 & 15,96 & 7,61 \\
\hline 9,69 & 10,41 & 7,41 & 9,93 & 2,39 & 9,96 & 2,78 \\
\hline 15,45 & 13,35 & 13,59 & 11,67 & 24,45 & 12,15 & 21,31 \\
\hline 13,35 & 16,59 & 24,26 & 16,32 & 22,23 & 14,82 & 10,99 \\
\hline 10,75 & 11,89 & 10,61 & 13,92 & 29,46 & 11,43 & 6,35 \\
\hline 12,19 & 12,07 & 1,00 & 12,45 & 2,13 & 11,40 & 6,52 \\
\hline 12,19 & 11,97 & 1,82 & 12,21 & 0,17 & 11,30 & 7,30 \\
\hline
\end{tabular}

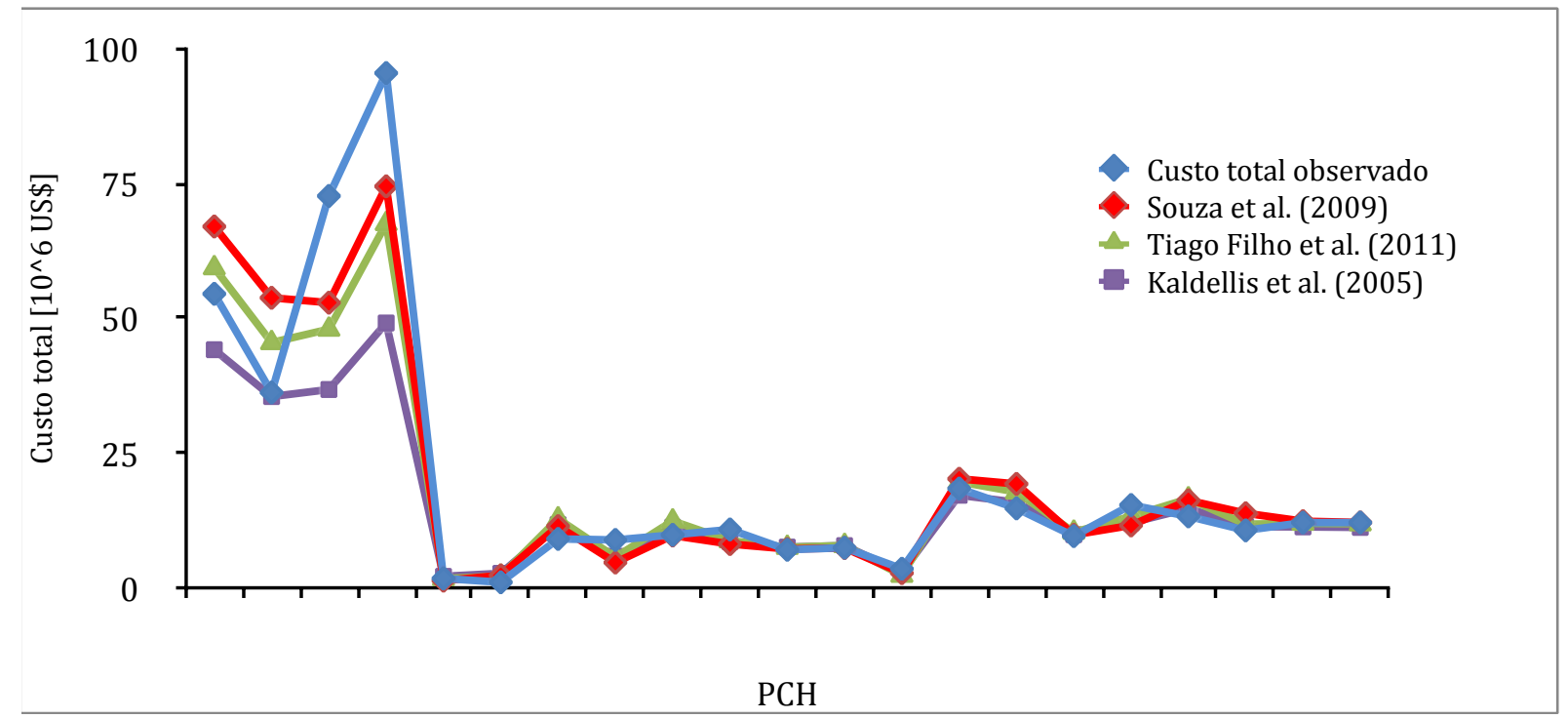

Figura 2. Comparação entre custos estimados e observados.

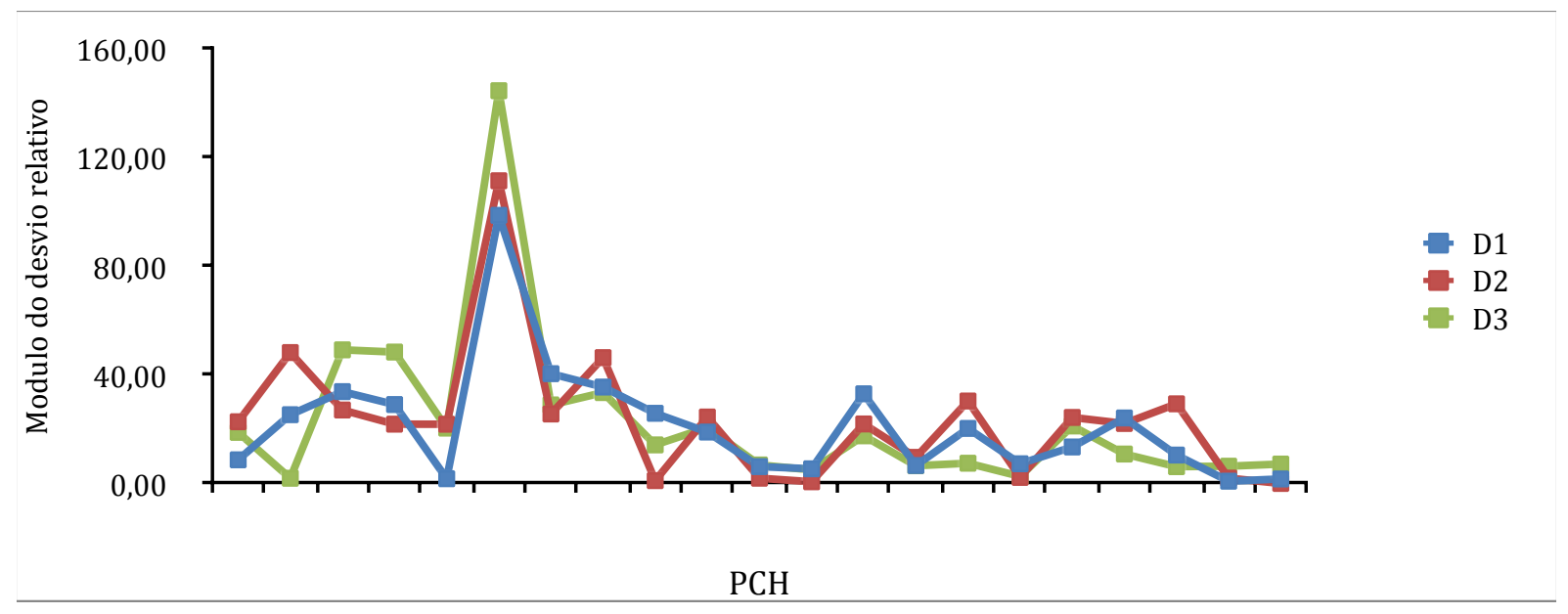

Figura 3. Desvios relativos (em módulo) de cada uma das estimativas e os valores reais.

SANTOS, I.F.S.; TIAGO FILHO, G.L.; BARROS, R.M.; LEMOS, H.. Ajuste e avaliação dos modelos agregados de estimativas de custo de PCHs no Brasil. Labor \& Engenho, Campinas [SP] Brasil, v.9, n.3, p.13-22, jul./set. 2015. http://www.conpadre.org 
Pela observação da Figura 3 pode-se concluir que os desvios relativos apresentam valores altos e insatisfatórios em um pico entre os pontos 5 e 9, mas sendo menores que $40 \%$ em grande parte dos projetos observados.

Já pela análise da Tabela 2, observamos que em oito pontos o método de Kaldellis et al. (2005) esteve mais próximo dos valores observados, enquanto que o método de Souza et al. (2009) esteve mais próximo sete vezes e o de Tiago Filho et al. (2011) seis. Contudo o método de Tiago Filho et al. (2011) foi aquele que possuiu o menor somatório de desvios (em módulo) considerando-se todos os pontos. Embora estas análises não sejam significativas ao ponto de permitir a conclusão de qual estimativa é a melhor para ser aplicada no cenário Brasileiro, estas nos permitem a conclusão de que a metodologia baseada no FA se aproxima melhor do cenário de custos Brasileiros em geral, embora não necessariamente se aproxime mais de cada projeto em particular.

Pode-se ainda por meio das Tabelas 1 e 2 calcular o custo unitário médio de cada estimativa e os desvios padrão entre os custos unitários, como apresenta a Tabela 3. Nesta observa-se que embora os custos unitários médios obtidos pelas 4 metodologias sejam bem próximos, o desvio padrão entre os custos observados em projetos reais é bem superior ao das estimativas. Este fato demonstra a heterogeneidade encontrada entre os diversos custos de PCHs, o que contribui para o aumento da dificuldade de parametrização destes custos. A estimativa que apresentou os custo unitário médio e o desvio padrão mais próximo dos dados reais foi a estimativa de Souza et al. (2009).

Tabela 3. Comparação dos custos unitários médios.

\begin{tabular}{|c|c|c|}
\hline Método & Cun médio [US\$/kW] & Desvio Padrão \\
\hline Custo observado & 2420,285 & 747,943 \\
\hline Tiago Filho et al. $(2011)$ & 2366,962 & 278,460 \\
\hline Souza et al. $(2009)$ & 2399,746 & 550,131 \\
\hline Kaldellis et al. $(2005)$ & 2221,722 & 271,917 \\
\hline
\end{tabular}

Como as estimativas de custo são importantes por serem fator limitante a previsão da rentabilidade dos empreendimentos, a seguir aplicou-se as três estimativas de custo as estimativas de benefícios de um projeto real de uma $\mathrm{PCH}$. O projeto em questão é referente a uma área localizada no rio Lourenço Velho (Minas Gerais, Brasil). Os estudos hidrológicos foram realizados em função das áreas de drenagem das estações hidrometeorológicas localizadas na sub-bacia na qual está localizado o Rio Lourenço Velho. A curva de permanência de vazões utilizada nos cálculos hidroenergéticos no local de implantação é apresentada na Figura 4.

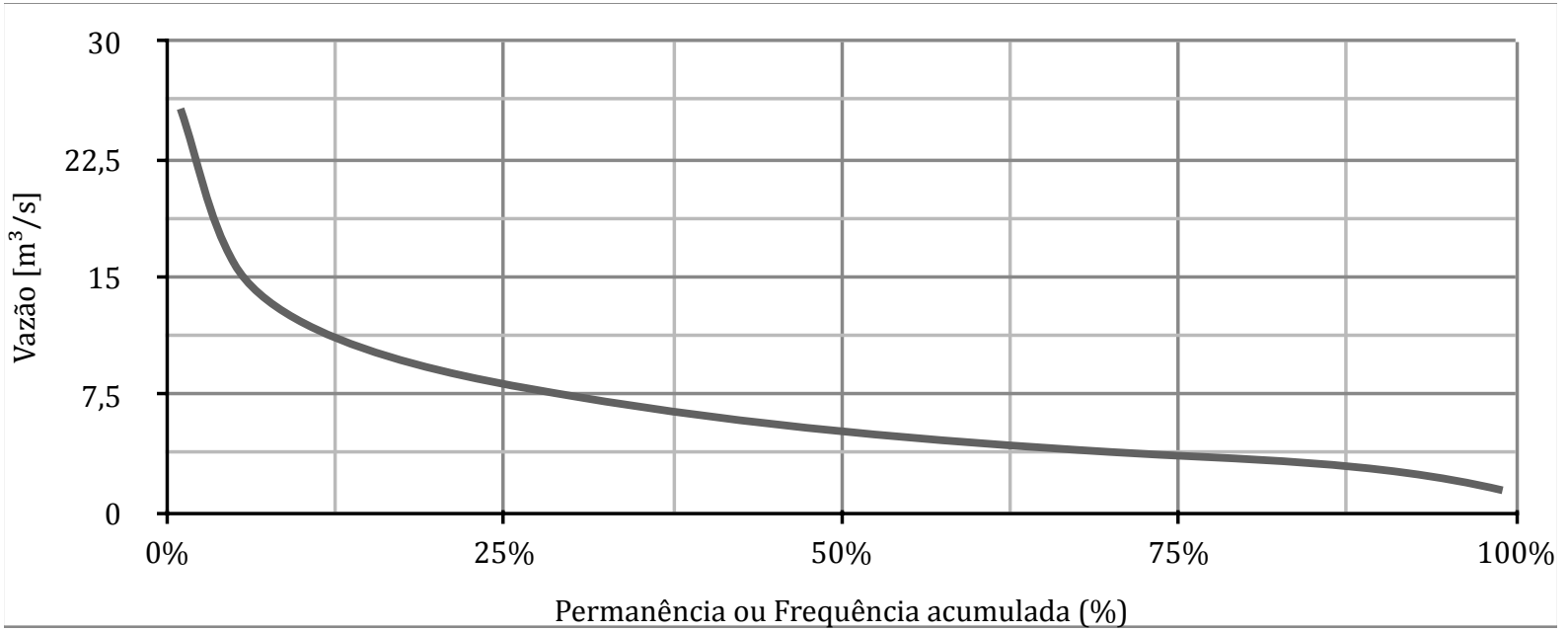

Figura 4. Curva de permanência do local de implantação da PCH.

SANTOS, I.F.S.; TIAGO FILHO, G.L.; BARROS, R.M.; LEMOS, H.. Ajuste e avaliação dos modelos agregados de estimativas de custo de PCHs no Brasil. Labor \& Engenho, Campinas [SP] Brasil, v.9, n.3, p.13-22, jul./set. 2015. http://www.conpadre.org 
A determinação da potência instalada na central foi realizada utilizando o método do máximo beneficio liquido (MBL). Este consiste em calcular os benefícios líquidos anuais (BL) gerados por cada vazão e cada freqüência e se verificar qual a vazão (potência) a ser implantada que o maximiza. De acordo com Cardoso et al. (2011), o valor de BL anual é calculado pela Equação 11.

$$
B L=E . T-P .(C u n . F R C+C o m)
$$

Sendo: $B L=$ beneficio liquido anual, $E=$ energia gerada anualmente $[\mathrm{KWh} / \mathrm{h}]$, $P=$ potência instalada, Cun = Custo unitário (calculado via estimativas anteriores), Com $=$ Custos de operação e manutenção, adotados $=5 \%$ do investimento inicial, conforme sugere Eletrobrás (2002), FRC = fator de recuperação do capital e $T=$ Valor da tarifa de energia elétrica $=60,92[\mathrm{US} \$ / \mathrm{kW}]$.

O Fator de recuperação de capital por sua vez foi calculado pela Equação 12. A vida útil adotada para as análises foi igual ao tempo de concessão: $\mathrm{n}=30$ anos, e a taxa de juros i foi adotada como sendo $=9 \%$.

$$
F R C=\frac{i \cdot(i+1)^{n}}{(i+1)^{n}-1}
$$

A Figura 5 apresenta as curvas de beneficio liquido obtidas com as três estimativas. Nela observamos que as estimativas de Souza et al. (2009) e Tiago Filho et al. (2011) resultam em curvas bem próximas, sendo a curva obtida pela estimativa de Kaldellis et al. (2005) mais distante se aproximando das outras estimativas conforme a potência aumentava.

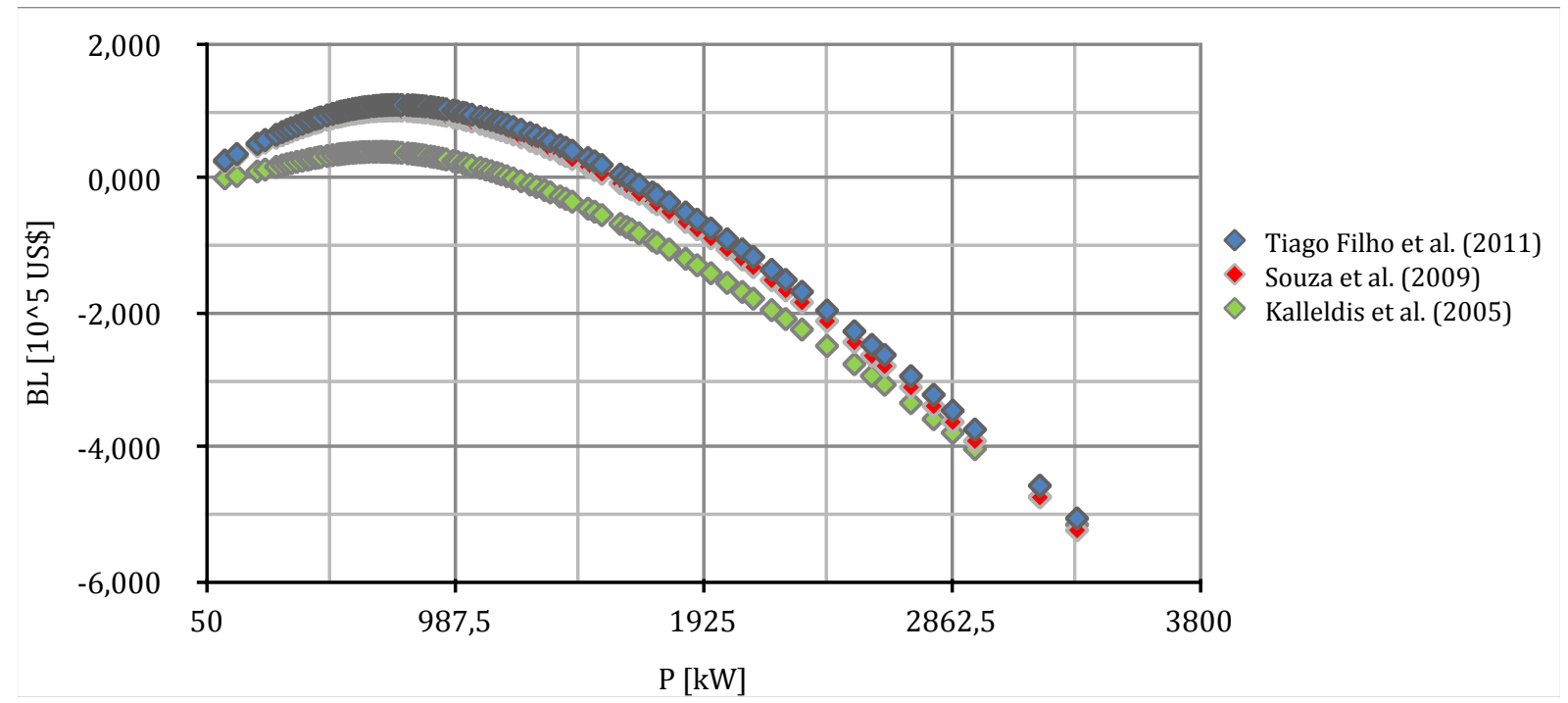

Figura 5. Curvas de Benefício Líquido.

A Tabela 4 abaixo apresenta ainda a comparação dos valores de importantes grandezas do projeto da PCH, quando está é operada no ponto de MBL, pelas três estimativas aqui estudadas. Na mesma tabela ainda está apresentada a tarifa de venda de energia que começava a tornar o empreendimento viável, para alguma das vazões disponíveis (Tviab). Pode-se observar que as diferenças entre as três estimativas são bem pequenas, sendo pouco intensificadas na estimativa de Kaldellis et al. (2005), com exceção dos valores de Tviab, muito superior nesta terceira estimativa (51 e 43 [\%] superior as outras Tviab da Tabela 4). Tal fato demonstra o impacto que uma estimativa de custo pode apresentar sobre os cálculos de viabilidade econômico-financeira do empreendimento, podendo ocasionalmente até indicar a inviabilidade de um empreendimento, quando na verdade, este é viável. 
Tabela 4. Resultados das estimativas no ponto de MBL.

\begin{tabular}{|c|c|c|c|c|}
\hline Estimativa & BL máximo [10 $0^{5}$ US\$] & P ótima [kW] & Q ótima $\left[\mathrm{m}^{3} / \mathrm{s}\right]$ & $\mathrm{T}_{\text {viab }}$ [US\$/MWh] \\
\hline Tiago & 2,61 & 771 & 3,25 & 34,87 \\
\hline Souza & 2,42 & 758,16 & 3,19 & 36,97 \\
\hline Kalleldis & 2,05 & 707,07 & 2,98 & 52,94 \\
\hline
\end{tabular}

\section{Conclusões}

No presente artigos foram comparadas três estimativas de custo aplicadas ao mercado hidrelétrico Brasileiro. Por meio dos resultados do presente trabalho pode-se concluir que a estimativa com menor somatório de desvios, quando comparada com os dados de projeto do cenário Brasileiro foi a desenvolvida por Tiago Filho et al. (2011), enquanto que o método de Kaldellis et al. (2005) resultou, por um maior número de vezes, em um custo observado mais próximo do real. Este fato demonstra que embora a abordagem de Tiago Filho se aproxime mais da tendência observada nos custos dos projetos em geral, esta não é a mais precisa em todos os pontos analisados.

Como as estimativas de custo são importantes para avaliação preliminar da viabilidade de um empreendimento em estudo, as três estimativas aqui listadas foram utilizadas para determinação das grandezas de um projeto real de $\mathrm{PCH}$, quando esta opera no ponto de máximo benefício liquido. As diferenças de benefícios obtidos pelos modelos de Tiago Filho et al. (2011) e Souza et al. (2009) foram bem pequenas em todas as potências analisadas, sendo os parâmetros obtidos no ponto ótimo também bem próximos em ambas modelagens.

A estimativa de Kaldellis et al. (2005) foi a mais distante das demais, especialmente quanto ao parâmetro Tviab (tarifa a partir da qual haveria viabilidade da central para qualquer uma das vazões possíveis de serem implantadas), que chegou a ser $50 \%$ superior as outras estimativas. Isto demonstra os impactos, sobre a análise de atratividade financeira da $\mathrm{PCH}$, de uma estimativa de custo, que embora se aproxime razoavelmente bem dos dados do cenário nacional, apresenta alto desvio para um projeto especifico. Uma forma de se minimizar este problema é através da construção de estimativas para regiões menores ou então da aplicação de certo modelo somente sobre projetos de regiões próximas aqueles que satisfatoriamente foram aproximados pelo modelo.

\section{Referências}

AGGIDIS, G. A. et al. The costs of small-scale hydro power production: Impact on the development of existing potential. Renewable Energy. Vol.35, p. 2632-2638. Ed. Elsevier, 2010.

ANEEL. Agência Nacional de Energia Elétrica, 2003. Resolução ANEEL no 652, de 9 de dezembro de 2003. Disponível em: http://www.aneel.gov.br/cedoc/res2003652.pdf. Acesso em: 24/02/2014.

CARDOSO, R. B. et al. Uma avaliação do método expedito para determinação da vazão de projeto em pequenas centrais hidrelétricas. Centro Nacional de Referência em Pequenas Centrais Hidrelétricas (CERPCH), 2011. Disponível em: http://www.cerpch.unifei.edu.br/arquivos/artigos/pch-shp-33-pag10a14.pdf. Acesso em 24/02/2014.

KALDELLIS, J.K. et al. Techno-economic evaluation of smallhydro power plants in Greece: a complete sensitivity analysis. Energy Policy, Vol.33, p.1969-85. Elsevier, 2005.

MASON, I. G., et al. 100\% renewable electricity generation system for New Zealand utilising hydro, wind, geothermal and biomass resources. Energy Policy, Vol. 38, 2010, p. 3973-3984.

SANTOS, A. M. H. et al. Estimação de custos de PCH. VII CBPE - Congresso Brasileiro de Planejamento Energético. São Paulo, 2010.

SANTOS, I.F.S.; TIAGO FILHO, G.L.; BARROS, R.M.; LEMOS, H.. Ajuste e avaliação dos modelos agregados de estimativas de custo de PCHs no Brasil. Labor \& Engenho, Campinas [SP] Brasil, v.9, n.3, p.13-22, jul./set. 2015.

http://www.conpadre.org 
SINGAL, S. K.; SAINI, R. P. Analytical approach for development of correlations for cost of canal-based SHP schemes. International Conference on Small Hydropower -Hydro Sri Lanka, 22-24, 2007.

SOUZA, Z. et al. Centrais Hidrelétricas: Implantação e Comissionamento. $2^{a}$ ed., Rio de Janeiro: Ed. Interciência, 2009.

TIAGO FILHO, G. L. "Viabilidade econômico financeiro do desenvolvimento de projetos de PCHs". VII Conferência de Centrais Hidrelétricas, Centro de Convenções do Novotel Center Norte, São Paulo, 03 e 04 ago. 2011.

TIAGO FILHO, G. L. et al. Uma abordagem para prospecção do custo unitário de PCH baseada no seu fator de aspecto. Revista Hidro \&Hydro: PCH Noticias e SHP News. Vol. 56, p. 50-55, 2013.

ZHANG, Q. F. et al. Small Hydropower Cost Reference Model. Oak Ridge National Laboratory. U.S. Department Of Energy, 2012. 